ARTICLE

\title{
Directed self-assembly of herbal small molecules into sustained release hydrogels for treating neural inflammation
}

Jun Zheng ${ }^{1}$, Rong Fan², Huiqiong Wư,3, Honghui Yao ${ }^{4}$, Yujie Yan ${ }^{4}$, Jiamiao Liu ${ }^{4}$, Lu Ran ${ }^{5}$, Zhifang Sun ${ }^{1}$, Lunzhao Yi ${ }^{5}$, Li Dang ${ }^{6}$, Pingping Gan ${ }^{7}$, Piao Zheng ${ }^{8}$, Tilong Yang ${ }^{9}$, Yi Zhang (1) ${ }^{1,2}$, Tao Tang ${ }^{2} \&$ Yang Wang (D) ${ }^{2}$

Self-assembling natural drug hydrogels formed without structural modification and able to act as carriers are of interest for biomedical applications. A lack of knowledge about natural drug gels limits there current application. Here, we report on rhein, a herbal natural product, which is directly self-assembled into hydrogels through noncovalent interactions. This hydrogel shows excellent stability, sustained release and reversible stimuli-responses. The hydrogel consists of a three-dimensional nanofiber network that prevents premature degradation. Moreover, it easily enters cells and binds to toll-like receptor 4 . This enables rhein hydrogels to significantly dephosphorylate $1 \kappa B \alpha$, inhibiting the nuclear translocation of p65 at the NFkB signalling pathway in lipopolysaccharide-induced BV2 microglia. Subsequently, rhein hydrogels alleviate neuroinflammation with a long-lasting effect and little cytotoxicity compared to the equivalent free-drug in vitro. This study highlights a direct self-assembly hydrogel from natural small molecule as a promising neuroinflammatory therapy.

\footnotetext{
${ }^{1}$ Key Laboratory of Hunan Province for Water Environment and Agriculture Product Safety, College of Chemistry and Chemical Engineering, Central South University, 410083 Changsha, China. ${ }^{2}$ Institute of Integrative Medicine, Key Laboratory of Hunan Province for Liver Manifestation of Traditional Chinese Medicine, Xiangya Hospital, Central South University, 410008 Changsha, China. ${ }^{3}$ Key Laboratry of Materials Processing and Mold, Ministry of Education, Zhengzhou University, 450002 Zhengzhou, China. ${ }^{4}$ Xiangya School of Medicine, Central South University, 410013 Changsha, China. ${ }^{5}$ Yunnan Food Safety Research Institute, Kunming University of Science and Technology, 650500 Kunming, China. ${ }^{6}$ Department of Chemistry and Key Laboratory for Preparation and Application of Ordered Structural Materials of Guangdong Province, Shantou University, 515063 Shantou, China. ${ }^{7}$ Department of Oncology, Xiangya Hospital, Central South University, 410008 Changsha, China. ${ }^{8}$ College of Integrated Traditional Chinese and Western Medicine, Hunan University of Chinese Medicine, 410208 Changsha, China. ${ }^{9}$ Southern University of Science and Technology, 518055 Shenzhen, China. These authors contributed equally: Jun Zheng, Rong Fan. Correspondence and requests for materials should be addressed to Y.W. (email: wangyang_xy87@csu.edu.cn) or to Y.Z. (email: yzhangcsu@csu.edu.cn) or to T.T. (email: tangtaotay@csu.edu.cn)
} 
$\mathrm{N}$ atural small molecules are regarded as promising drug resources due to their wide range of pharmacophores and high degrees of stereochemistry ${ }^{1,2}$. Unfortunately, clinical treatments using these well-studied natural products are limited owing to the poor solubility and unsatisfying stability 3,4 . For years, scientists focus on hydrogel-based drug delivery systems to improve the solubility and stability of natural small molecules ${ }^{5,6}$. Numerous supramolecular hydrogels containing drug complexes have successfully been developed in the laboratory for inflammatory alleviation 7,8 , wound repair ${ }^{9}$, bacteria resistance, and tumor inhibition ${ }^{10,11}$. However, clinical researchers continually find that the involvement of drug carriers may lead to poor biocompatibility and biodegradability, low loading efficacy, and potential side effects. Additionally, these biological materials from drug carriers require complex syntheses and are relatively expensive ${ }^{12}$, which create great obstacles for effective clinical application. Thus, chemists and pharmacologists persistently desire to prepare directed self-assemble hydrogels, referring to self-delivering, self-releasing, stable, injectable and stimuliresponsive hydrogels without any structural modification and delivery cargoes. The hydrogels are expected to be capable of superior solubility, optimal therapeutic efficacy, and almost no cytotoxicity.

Abundant innovative efforts on the direct self-assembly biological hydrogels of small molecules (especially lanreotide, diphenylalanine, Fmoc-diphenylalanine, and curcumin) have been explored ${ }^{13-15}$. Many self-assembly hydrogel systems derived from natural products following structural modification have been invented (such as taxol ${ }^{16,17}$, camptothecin ${ }^{18}$, and dexamethasone ${ }^{19}$ ). Despite these endeavors, designing directed selfassemble hydrogels formed by natural small molecules is still a formidable challenge. The exploration still largely relies on serendipitous, because the construction requires a perfectly stable equilibrium among a series of complicated and meticulous balances, including amphipathicity, intermolecular interactions, chirality, and spatial molecular arrangement ${ }^{20}$.

We have been continuously working on rhein (the chemical structure is shown in Fig. 1a), an anthraquinone mainly isolated from the traditional Chinese medicine rhubarb (Rheum palmatum L. or Rheumtanguticum Maxim, Dahuang in Chinese) for at least a decade ${ }^{21}$. Rhein performs neuroprotection via antiinflammation in treating cerebral injuries including neurodegenerative diseases and traumatic brain injury ${ }^{22,23}$. Nevertheless, the solubility of rhein remains poor and simultaneously exhibits low bioavailability by metabolism of glucuronidation in liver ${ }^{24,25}$, resulting in a hindrance to clinical transformation. To enhance the therapeutic efficacy and minimize negative effects, a few efforts to prepare polymeric microparticles and nanoparticles containing rhein have been attempted ${ }^{26,27}$. However, drug loss during the fabrication process and premature release of payload still lead to lower drug loading and adverse systemic toxicity ${ }^{3}$.

We believe that directed self-assembly of rhein should be a promising solution. In this study, rhein directly self-assembles into a supramolecular hydrogel via intermolecular $\pi-\pi$ interactions and hydrogen bonds. The rhein hydrogel has excellent biostability, sustained drug release, and reversible stimuli-responsive performances. In particular, the as-prepared rhein hydrogel exerts better antineuroinflammation than its free-drug form with almost no cytotoxicity. To investigate such superior anti-neuroinflammatory effects, we explore the underlying molecular mechanisms. We demonstrate that the rhein supramolecular hydrogel is easier to enter cells than free-drugs, and increases accumulation to intensively bind to the active site of toll-like receptor 4 (TLR4). These properties achieve optimal anti-inflammation through inhibition of the TLR4/ $\mathrm{NF} K \mathrm{~B}$ signaling pathway, which essentially boosts the therapeutic efficacy and reduces the negative effects. These features trigger the rhein hydrogel to serve as a promising therapeutic agent for antineuroinflammation.

\section{Results}

Morphology and gelation properties of rhein hydrogel. In this study, the rhein direct self-assembly hydrogel was formed in PBS buffer by simple ultrasound with heating to obtain a homogeneous solution and subsequently cooled to room temperature. The minimum gelation concentration was $14.1 \mathrm{mM}$ (Supplementary Fig. 1). The appearance of the hydrogel was a uniform orange-red. The scanning electron microscopy (SEM) images revealed that the scaffold was a $3 \mathrm{D}$ network composing of nanofibers (Fig. 1b). As can be observed in the transmission electron microscopy (TEM) and atomic force microscope (AFM) images, the nanofibers had an average diameter of approximately $30 \mathrm{~nm}$ with several micrometers in length (Fig. 1c and Supplementary Fig. 2).

Experimental tests indicated that $\mathrm{pH}$ intensively influenced the direct self-assembly process. The optimal $\mathrm{pH}$ values of the gel were between 8.0 and 9.4 (Supplementary Fig. 3a). Under this environment, we produced a translucent hydrogel. When the value of $\mathrm{pH}$ was above 9.4, the hydrogel began to collapse and became a blood red solution. The SEM images confirmed that the solution possessed a short, ribbon-like structure (Supplementary Fig. $3 \mathrm{~b}$ ). Within the ranges between $\mathrm{pH} 8.0$ and $\mathrm{pH} 6.8$, the sample was a viscous gel rather than a translucent gel. The microscopic morphology predominantly exhibited long fibers, while there were several short fibers distributed on the surface (Supplementary Fig. 3c). Once the pH values were set under 6.8, we observed precipitate formation. The SEM images displayed that these precipitates were the short, rod-like-structures (Supplementary Fig. 3d). These results suggest that the formation of the hydrogel is highly dependent on the degree of carboxyl deprotonation of rhein molecules. To evaluate the role of the carboxyl group in gel formation, we sought to prepare the other anthraquinones from rhubarb, including emodin, chrysophanol, aloe-emodin, and physcion (Supplementary Fig. 4). We found that none of these anthraquinones formed hydrogels under the conditions tested, demonstrating that the carboxyl group plays an important role in the gelation process.

The rhein hydrogel showed multi-responsiveness to external environment changes (Fig. 1d). When the temperature increased to $40^{\circ} \mathrm{C}$, the hydrogel turned into solution. Once the temperature was adjusted to room temperature, the sol-to-gel phase transition occurred after several hours. In addition, the gel-sol transitions were triggered through vigorous shaking by hand ${ }^{28,29}$, and the gel state was restored after standing within several minutes. Furthermore, the reversible properties of the rhein hydrogel were investigated by oscillatory shear rheology. When the strain exceeded $23.74 \%$, the loss modulus $\left(\mathrm{G}^{\prime \prime}\right)$ was higher than the storage modulus $\left(\mathrm{G}^{\prime}\right)$, revealing the transition from the gel to the solution state (solution state: $G^{\prime}<G^{\prime \prime}$, gel state: $G^{\prime}>G^{\prime \prime}$ ) (Fig. 1e). The step-strain test (Fig. 1f) revealed that $G^{\prime}$ was higher than $G^{\prime \prime}$ at low strain $0.1 \%$, and $G^{\prime}$ was lower than $G^{\prime \prime}$ at higher strain $35 \%$. The material properties of the rhein hydrogel recovered when transitioning from high strain to low strain, displaying the injectable and self-healing properties of the rhein hydrogel ${ }^{30,31}$. We also performed rotated rheology measurement to examine the viscoelastic properties. The dynamic frequency sweep showed that the values of $G^{\prime}$ were 5 times larger than those of $G^{\prime \prime}$ (Fig. 1g). Moreover, there was a weak dependency with frequency. Additionally, the $G^{\prime}$ was much greater than the $G^{\prime \prime}$ during the entire process, according to the dynamic time sweep data (Fig. 1h) 32. The excellent thixotropy and thermo-reversibility indicate that the rhein hydrogel can serve as an injectable hydrogel ${ }^{33}$. 
a<smiles>O=C(O)c1cc(O)c2c(c1)C(=O)c1cccc(O)c1C2=O</smiles>
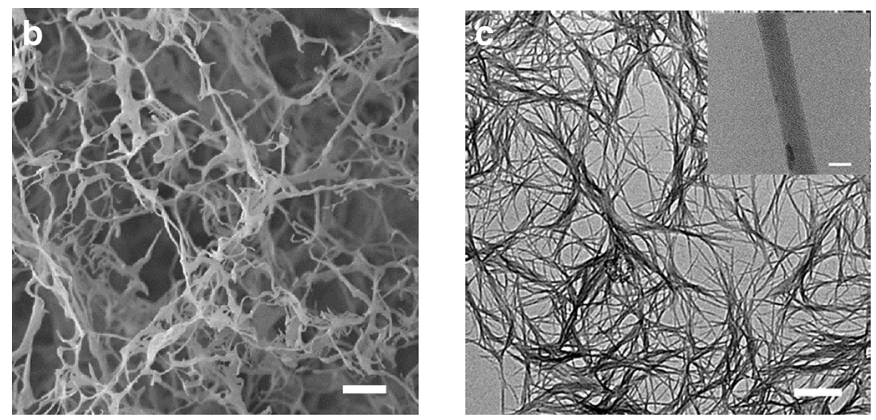

d
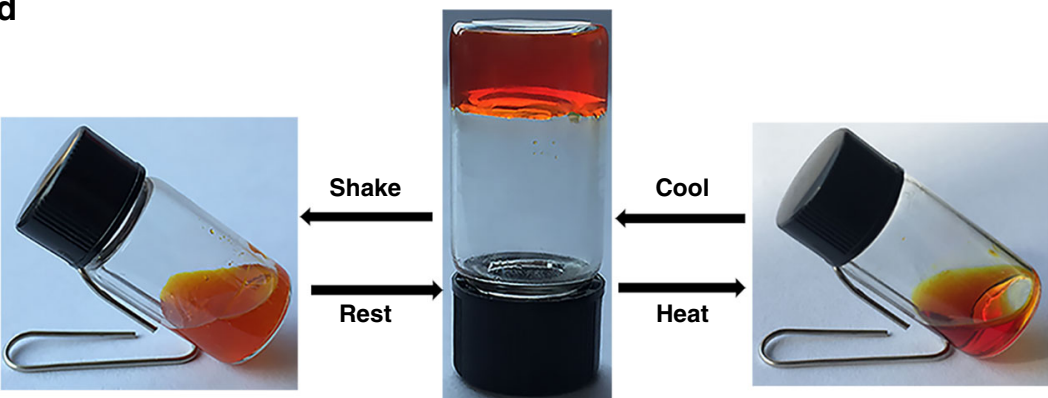

e

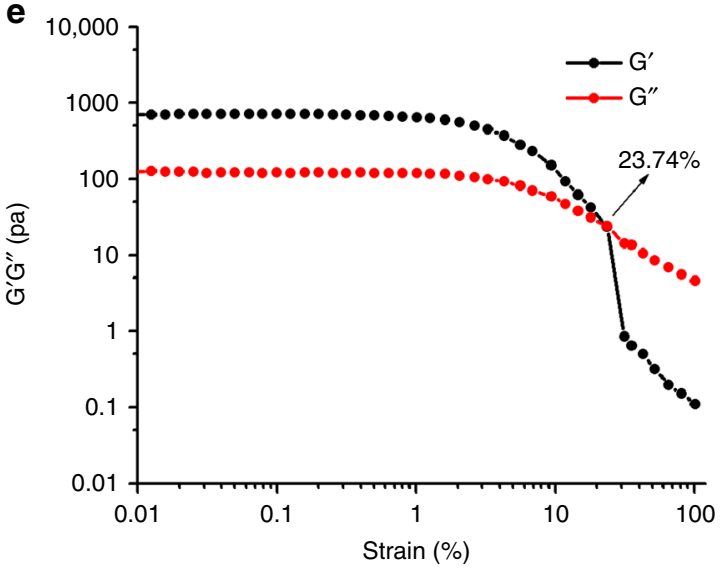

g

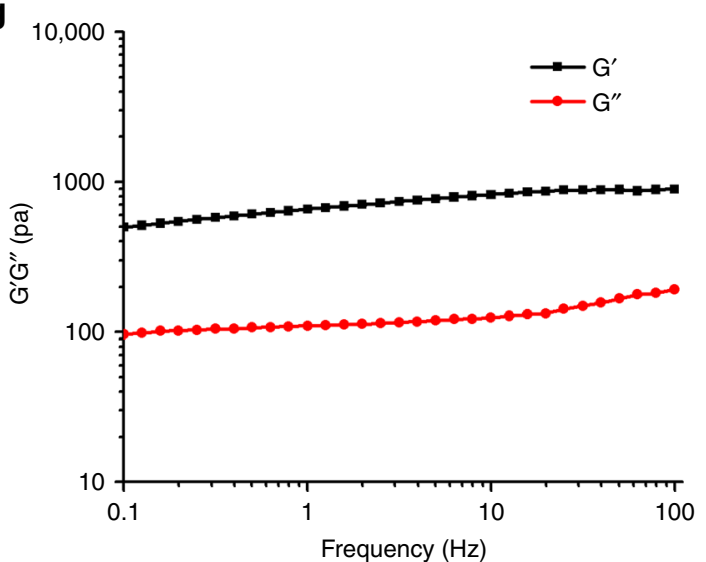

$\mathbf{f}$

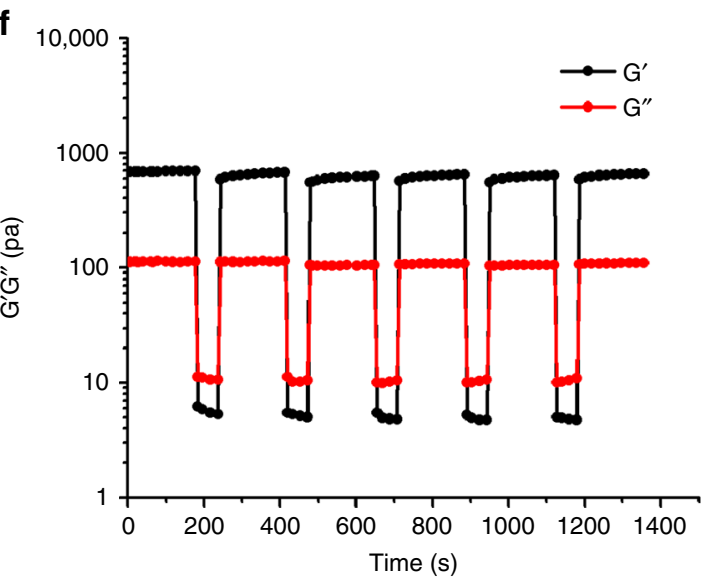

h



Fig. 1 Molecular formula of rhein and characterization of the rhein hydrogel. a Chemical structures of rhein and digital images of the rhein hydrogel. $\mathbf{b}$ SEM image of the rhein hydrogel, scale bar: $2 \mu \mathrm{m}$. c TEM image of the rhein hydrogel, scale bar: $500 \mathrm{~nm}$. The upper right is a partial enlarged view, scale bar: $20 \mathrm{~nm}$. d Reversible gel-sol transitions of rhein hydrogel triggered by shear stress and temperature. e Strain-dependent oscillatory shear rheology of the rhein hydrogel with a fixed frequency of $10 \mathrm{rad} \mathrm{s}^{-1}$. f Step-strain measurements of the hydrogel over five cycles at low strain (0.1\%) and high strain (35\%), frequency $10 \mathrm{rad} \mathrm{s}^{-1}$. $\mathbf{g}$ Dynamic frequency sweep of the rhein hydrogel, measured at $0.1 \%$ strain. $\mathbf{h}$ Dynamic time sweep of the rhein hydrogel, at the strain of $0.1 \%$ and at the frequency of $10 \mathrm{rad} \mathrm{s}^{-1}$. The concentration of hydrogel was $5 \mathrm{mg} \mathrm{mL}^{-1}(17.6 \mathrm{mM}), \mathrm{T}=25^{\circ} \mathrm{C}$ 
The directly self-assemble mechanism of rhein hydrogel. we used the mass spectrometry (MS) system to explore the structure of rhein aggregates, which is a powerful tool to investigate the assembly of aggregates ${ }^{34}$. The monomers $(\mathrm{m} / z$ 283.0249), dimers $(\mathrm{m} / z$ 589.0388), trimers $(\mathrm{m} / \mathrm{z}$ 895.0514), tetramers $(\mathrm{m} / \mathrm{z}$ 1201.0647) and higher-order aggregates were observed from MS (Fig. 2a, b). These aggregates existed as sodium salt clusters, the dimers were the predominant aggregate ions, indicating that the dimer is relatively stable relative to other aggregates. These results were further proved by the MS/MS analysis. We found the fragment ions of the dimer, rhein sodium salt $(\mathrm{m} / \mathrm{z}$ 305.0061) and rhein monomer from the MS/MS spectrum of higher-order aggregates (Fig. $2 \mathrm{c}-\mathrm{g}$ ). These results demonstrate that rhein monomers and sodium salt of rhein are the building block of rhein nanofibers ${ }^{35}$.

To shed light on the self-assembly process of rhein fibrils, we implemented spectroscopy studies. The FT-IR results suggested that there was a typical hydrogen bond absorption peak of $\mathrm{O}-\mathrm{H}$ at $3436 \mathrm{~cm}^{-1}$. During the gelation, the peak of O-H at $3436 \mathrm{~cm}^{-1}$ shifted to $3429 \mathrm{~cm}^{-1}$, simultaneously accompanied by an enhanced intensity in the hydrogel phase. On the other hand, the peak of $\mathrm{C}=\mathrm{O}$ at $1704 \mathrm{~cm}^{-1}$ shifted to $1662 \mathrm{~cm}^{-1}$ (Supplementary Fig. 5). These shifts indicate that hydrogen bonds are involved in the self-assembly process ${ }^{36,37}$.

The concentration-dependent UV/Vis absorption study displayed the characteristic anthraquinone group peak, which underwent a redshift from 418 to $503 \mathrm{~nm}$ as the concentration of rhein increased (Fig. 3a), which was assigned to the $\pi-\pi$ interactions of the anthraquinone structure. This phenomenon suggests that the aggregate configuration changed from H-type aggregation to J-type aggregation ${ }^{38-40}$.

We next performed fluorescence spectroscopy analysis to assess whether $\pi-\pi$ stacking played a role during the self-assembly process. As shown in Fig. 3b, an emission peak centered at 601 $\mathrm{nm}$ appeared in the solution, while the center of the emission peaks from the rhein hydrogel at 601 shifted to $616 \mathrm{~nm}$. As the concentration of the hydrogel increased, the fluorescence intensity decreased and the peak red shifted (Supplementary Fig. 6). The fluorescence tended to quench due to the aggregation of rhein molecules. The peak redshift was attributed to the presence of the anthraquinone excimert which mainly contributed to the self-assemble process through $\pi-\pi$ interactions ${ }^{39,41}$. Xray diffraction $(\mathrm{XRD})$ also confirmed this result. The apparent peak at $d=3.4 \AA$ was a typical distance of $\pi-\pi$ stacking interactions between the two moleculars (Fig. 3c) ${ }^{42}$.

We further employed circular dichroism (CD) spectra to explore the molecular packing of rhein within the hydrogel. The CD spectra exhibited strong negative peaks at $228 \mathrm{~nm}$ and 412 $\mathrm{nm}$ (Fig. 3d). These observations suggest that the chiral packing of rhein within the hydrogel is formed in the self-assembly process. The negative peaks revealed the left-hand helical arrangement. With the concentration of rhein hydrogel increased, the $\mathrm{CD}$ spectra displayed a strong negative bisignate $\mathrm{CD}$ signal between 210 and $300 \mathrm{~nm}$. These CD signal peaks showed an obvious redshift. The shift phenomena show that the Jaggregation of rhein occurs in the rhein hydrogel ${ }^{43}$.

Combining experimental results with theoretical calculation, we proposed a possible self-assembly process of rhein hydrogel. The gelation of rhein was highly dependent on $\mathrm{pH}$. When the $\mathrm{pH}$ value was set between 8.0 and 9.4, the rhein hydrogel was obtained. Under this condition, certain molecules were deprotonated to form rhein sodium salt. Rhein monomer and rhein sodium salt were together assembled to form dimer and higherorder aggregates. The two molecules were arranged in a J-type aggregation manner by $\pi-\pi$ stacking and hydrogel bonding to form a dimer. Due to the electrostatic repulsion between the carboxylic acid ions, two molecules tended to be arranged in opposite direction. The density functional theory (DFT) calculations also supported this conformation (Supplementary Fig. 7). Subsequently, dimers were further assembled into trimers, tetramers and higher-order aggregates. The rhein molecules continually added to the per-existing aggregates in a left helix fashion, resulting in the formation of the nanofiber with a lefthanded helical configuration. The nanofibers further crosslinked to form 3D networks (Fig. 3e).

Assessments of sustained release and cytotoxicity. We examined the sustained release of rhein hydrogel within a series of concentrations. Figure 4a confirmed the release profile. There was a fast release during the first $12 \mathrm{~h}$, and then the release of rhein showed a gradual process. Regardless of high concentration or low concentration, the release rate was up to $70 \%$ after $24 \mathrm{~h}$, which was attributed the good solubility of the hydrogel and the brittleness of the fibers ${ }^{7}$. As the gel concentration increased, the release rate decreased, accompanied by the extension of the release time. Moreover, the cumulative release percentage at low concentrations reached $90 \%$ within $36 \mathrm{~h}$. Only a small amount of rhein was released from 36 to $72 \mathrm{~h}$. The cumulative release rate under high concentrations merely came to $80 \%$ within $36 \mathrm{~h}$ and the release rate reached $88 \%$ after sustained released to $72 \mathrm{~h}$. The higher concentration of rhein hydrogel which possessed a closer fibers network, resulted in more durable release of the rhein molecules from hydrogel. These properties facilitate the rhein hydrogel to serve as a potential biomedical application.

Stability is a key indicator to evaluate whether a drug can potentially be used in clinical application. The rhein hydrogel maintained a good stability after being stored at room temperature for 3 months (Supplementary Fig. 8). In order to investigate the stability of the rhein hydrogel in cells. We used liquid chromatography-mass spectrometry (LC-MS) to detect the concentration of rhein in the BV2 cells treated with rhein hydrogel and free-drug for $3 \mathrm{~h}, 12 \mathrm{~h}, 24 \mathrm{~h}, 48 \mathrm{~h}$, and $72 \mathrm{~h}$, respectively. As shown in Fig. $4 \mathrm{~b}$, the concentration of the rhein from hydrogel group was higher than the free-drug group at each time point. Furthermore, the dimers were merely observed in rhein hydrogel group (Supplementary Fig. 9). These data suggest that rhein nanofiber is more likely to penetrate into cells than free-drug form. In addition, we found that the concentration of free-drug reached the top at $24 \mathrm{~h}$ and then decreased at $48 \mathrm{~h}$. While there was a plateau during $24-48 \mathrm{~h}$ in rhein hydrogel group, indicating that the rhein hydrogel is more stable in the cells.

To evaluate the toxicity, we executed the 3-(4,5-dimethylthiazol-2-yl)-2,5-diphenyltetrazolium bromide (MTT) assay by assessing the effect of the drug concentration on cell viability. Figure $4 \mathrm{c}$ suggested that the rhein hydrogel and the equivalent free-drug dosing showed no obvious cytotoxic effects on BV2 cells within the ranges of 5-40 $\mu \mathrm{M}$ at $24 \mathrm{~h}$. When the treatment continued to $48 \mathrm{~h}$ (Fig. $4 \mathrm{~d}$ ), $40 \mu \mathrm{M}$ of the free-drug significantly diminished the BV2 cells viability by $22 \%$ compared with the rhein hydrogel. Interestingly, the rhein hydrogel at various concentrations did not affect the viability of BV2 cells. The results demonstrate that the cytotoxicity of the rhein hydrogel is lower than that of the free-drug during the slow and sustained release ${ }^{44}$.

Rhein hydrogel exerts better anti-neuroinflammation. For medical transformation, we used a LPS-induced BV2 cell line as an in vitro model to estimate the anti-neuroinflammatory effects of the rhein hydrogel. During neuroinflammation, brain microglia underwent morphological changes and proliferation, triggering the excess production of various proinflammatory 
a
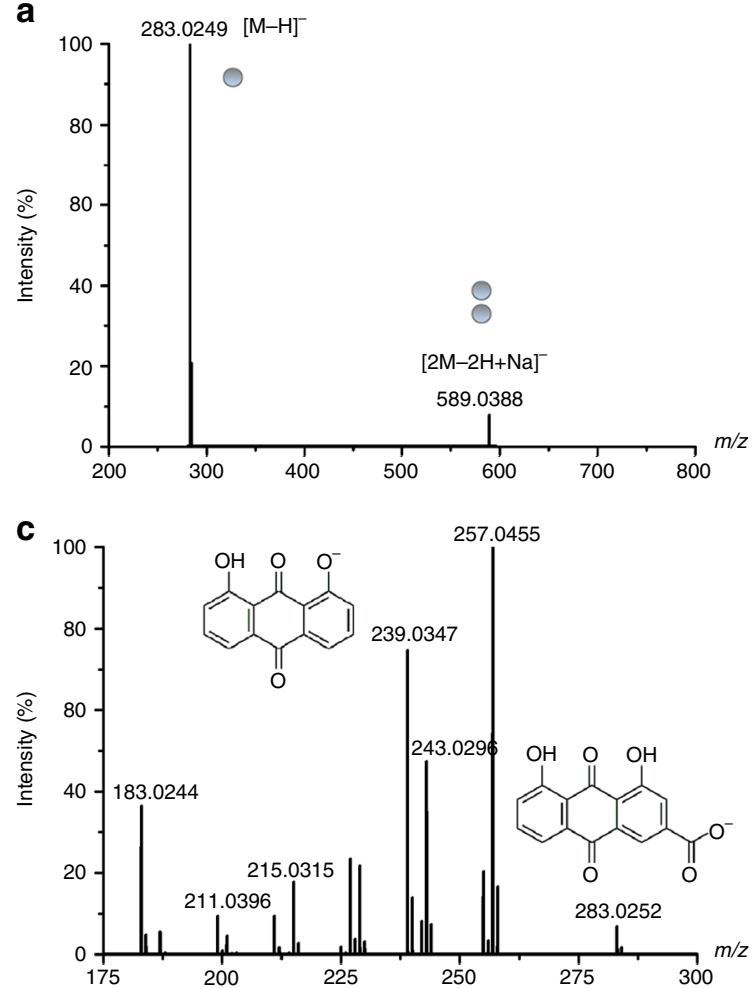

e

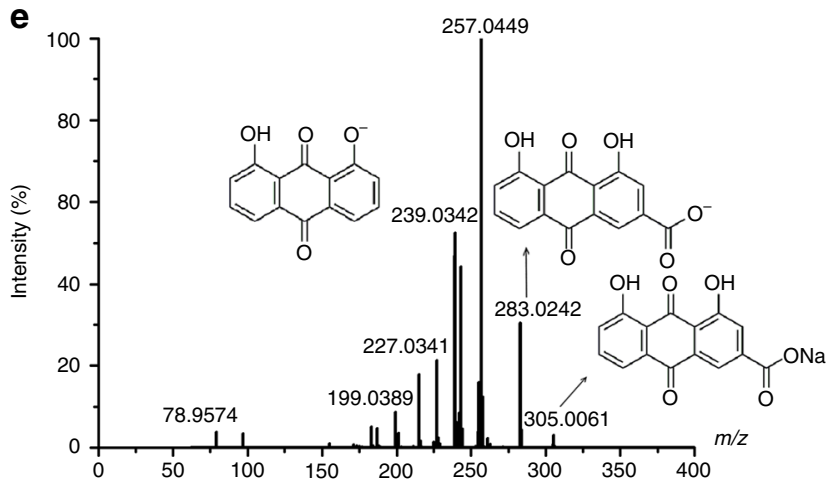

b

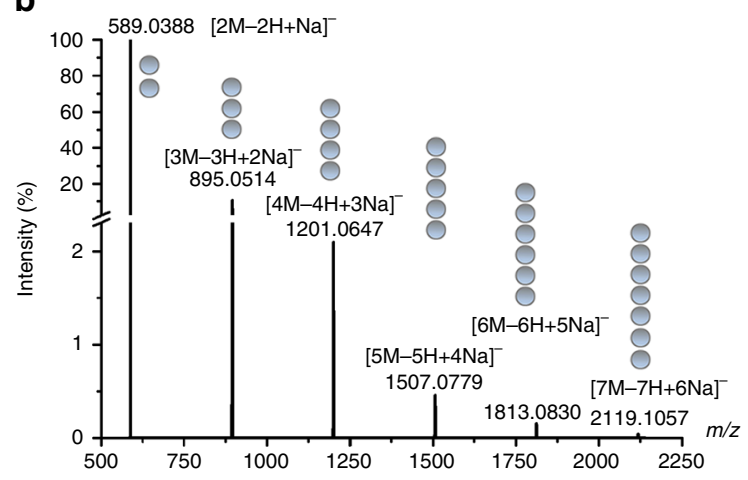

d
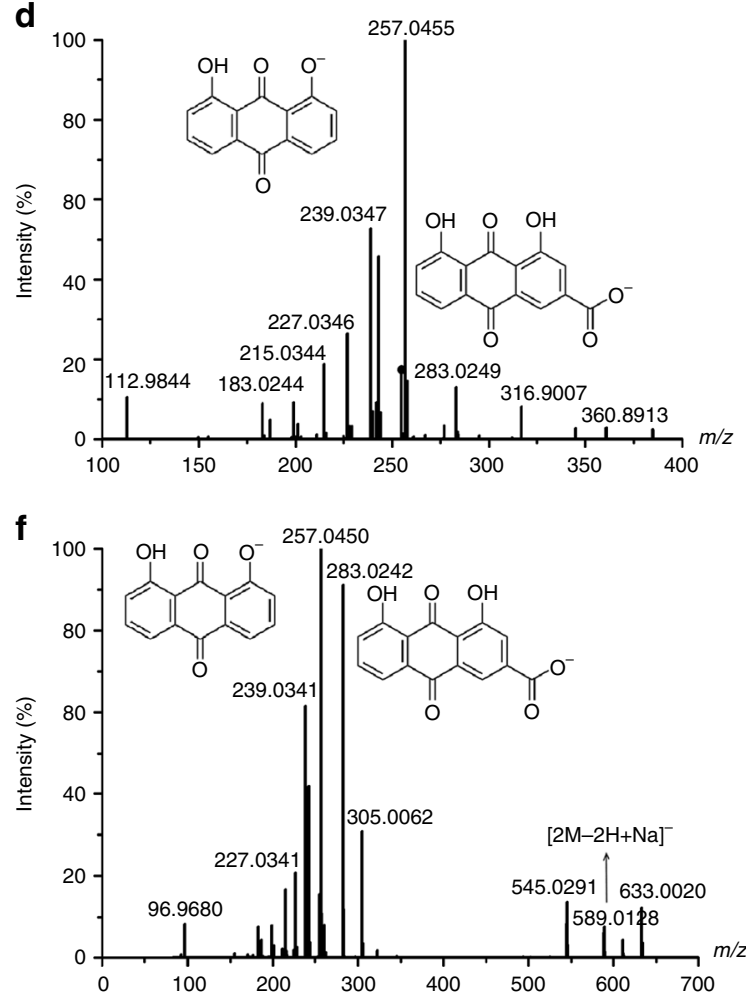

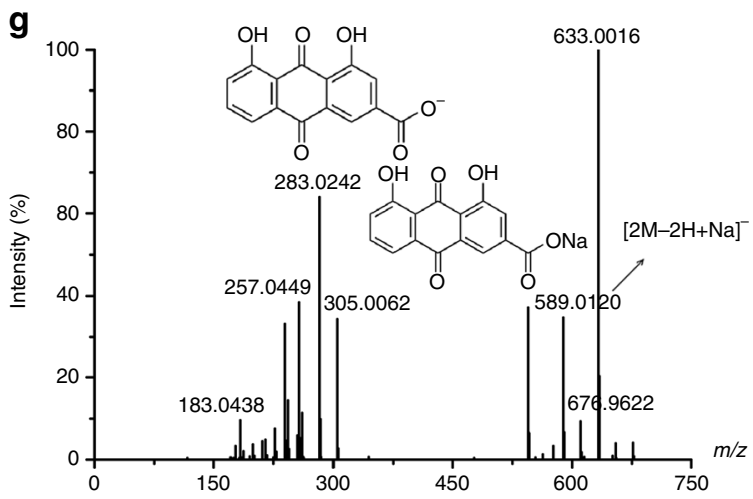

Fig. 2 Mass spectrometric analysis of the rhein hydrogel. a, b MS analyses of the rhein hydrogel. c MS/MS analyses of monomer $\left[M-\mathrm{H}^{-}\right.$. $\mathbf{d} \mathrm{MS} / \mathrm{MS}$ analyses of dimer $[2 \mathrm{M}-2 \mathrm{H}+\mathrm{Na}]^{-}$. e MS/MS analyses of trimer $[3 \mathrm{M}-3 \mathrm{H}+2 \mathrm{Na}]^{-}$. $\mathbf{f ~ M S / M S}$ analyses of tetramer $[4 \mathrm{M}-4 \mathrm{H}+3 \mathrm{Na}]^{-} . \mathbf{g} \mathrm{MS} / \mathrm{MS}$ analyses of pentamer $[5 \mathrm{M}-5 \mathrm{H}+4 \mathrm{Na}]^{-}$

mediators. TNF- $\alpha$ and IL- $1 \beta$ have been recognized as the key factors in neuroinflammation. Microglia cells are primed by neuroinflammatory disorders to produce exaggerated responses to IL- $1 \beta$ and TNF- $\alpha^{45}$. Thus, we primarily focused on the drug effects on these two cytokines. We tested the rhein hydrogel in a therapeutic setting in LPS-stimulated BV2 cells. LPS led to the marked accumulation of TNF- $\alpha$ and IL-1 $\beta$ compared to the control (Fig. 5). After $24 \mathrm{~h}$ treatment, the hydrogels and the 

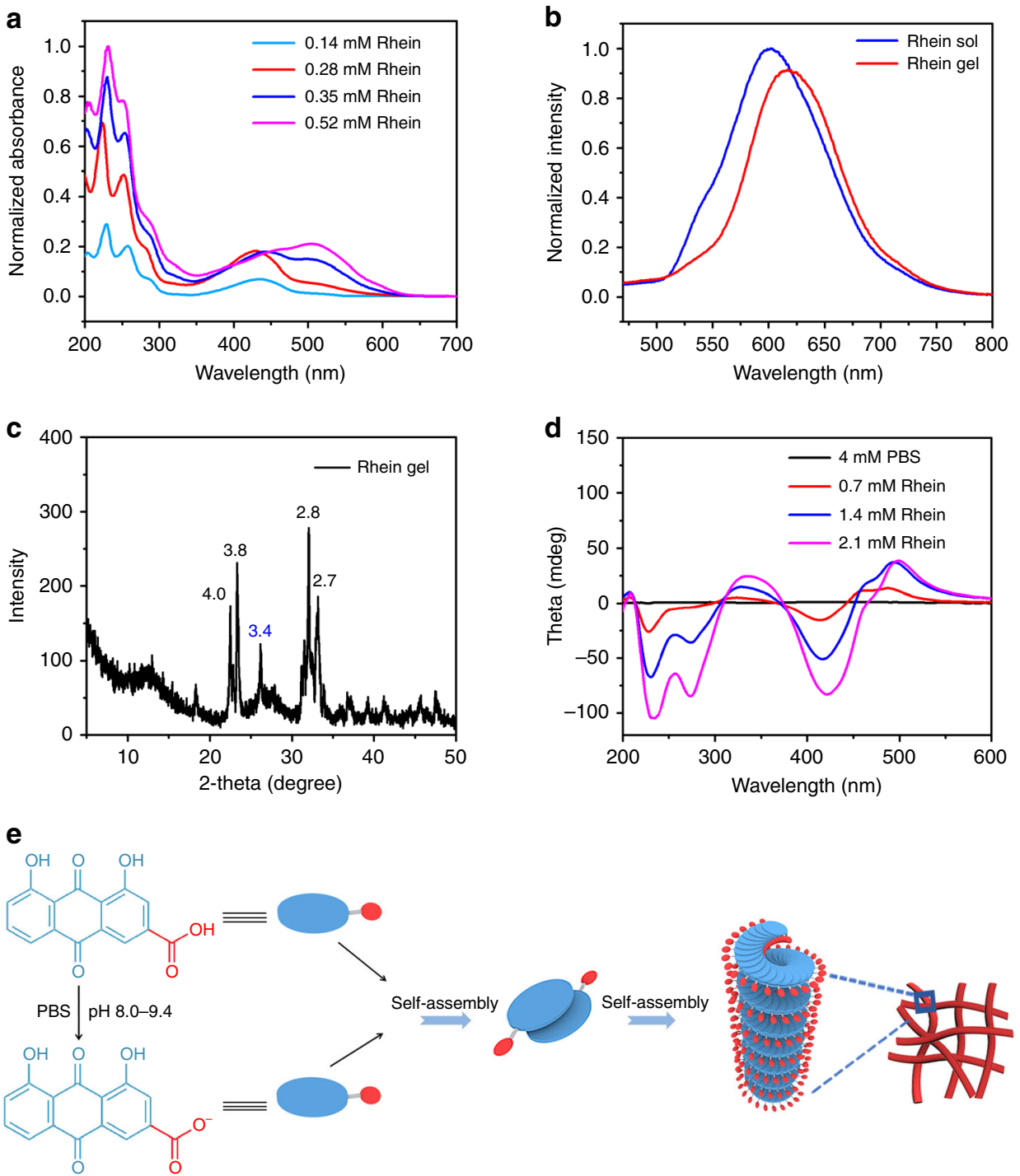

Fig. 3 The self-assemble mechanism of the rhein hydrogel. a UV/Vis spectrum of the rhein hydrogel. $\mathbf{b}$ Fluorescence emission spectra of rhein solution and hydrogel. The samples were recorded at $4 \mathrm{mg} \mathrm{mL}^{-1}(14.1 \mathrm{mM})$. c XRD pattern of the rhein xerogel (the unit of inserted distances is $\AA$ ). $\mathbf{d} C D$ spectra of the rhein hydrogel at different concentrations. e Self-assembly process diagram of the rhein hydrogel

equivalent free-drug dosing resulted in the obvious elimination of TNF- $\alpha$ and IL- $1 \beta$, as confirmed by the hypointensity of red (TNF- $\alpha$ ) and green (IL-1 $\beta$ ) fluorescent protein-positive cells coexpressed in the microglia kytoplasm (Fig. 5a). Similarly, subsequent western blotting and enzyme linked immunosorbent assay (ELISA) revealed that the levels of TNF- $\alpha$ and IL-1 $\beta$ were dramatically reduced with both drugs compared to the LPStreated group (Fig. 5b-f). In addition, the results of ELISA assays showed that both drugs notably decreased the releases of IL-6, IL12 , and iNOS compared to the LPS-treated group (Fig. $5 \mathrm{~g}-\mathrm{i}$ ).

To observe the sustained release efficacy, the treatment continued for $48 \mathrm{~h}$. We found that the hydrogel significantly lowered the levels of TNF- $\alpha$, IL-6, IL-12, and iNOS compared with the equivalent free-drug dosing. (Fig. 5). The observations reveal that the rhein hydrogel exhibits better neuroinflammatory prevention than the free-drug through the direct self-assembly nanofibers under sustained release control.

Rhein hydrogel enhances TLR4/NFkB inhibition. To further elucidate the anti-inflammatory pathways of the rhein hydrogel, it is critical to explore the underlying molecular mechanisms.
Neuroinflammation is mainly implicated in the activation of $\mathrm{p} 38, \mathrm{PI} 3 \mathrm{~K} / \mathrm{Akt}$ and TLR4/NFkB signaling pathways ${ }^{46-48}$. We used western blotting analyses to investigate the antineuroinflammatory mechanisms of rhein hydrogel and the free drug. We studied the effects of rhein and rhein hydrogel on TLR4/NFkB signaling pathways. Following activation of BV2 cells by LPS, increasing TLR4 expression followed by the enhancement of phosphorylation and degradation of $\mathrm{I} \kappa \mathrm{Ba}$ in the cytoplasmic extracts were observed compared to the control (Fig. 6b-e). NFkB activity was evaluated according to the nuclear translocation of the $\mathrm{p} 65$ subunit of NFKB. The findings revealed that LPS induced phosphorylation of I $\mathrm{B} a$, further resulting in the remarkable shift of $N F \kappa B$ p65 into the nucleus. The presence of rhein hydrogel significantly prevented increases in TLR4, phosphorylation of $\mathrm{I} \kappa \mathrm{Ba}$ and nuclear translocation of $\mathrm{p} 65$. In particular, during the phase of sustained release $(48 \mathrm{~h})$, the rhein self-assembly hydrogels better inhibited the TLR4/NFKB pathway than did the equivalent free-drug dosing (Fig. 6b-e). We also surveyed the effects of the rhein hydrogels and free-drug on the other neuroinflammatory signaling pathways, including p38 and PI3K/Akt (Supplementary Figs. 10 and 11). The results indicate that no 

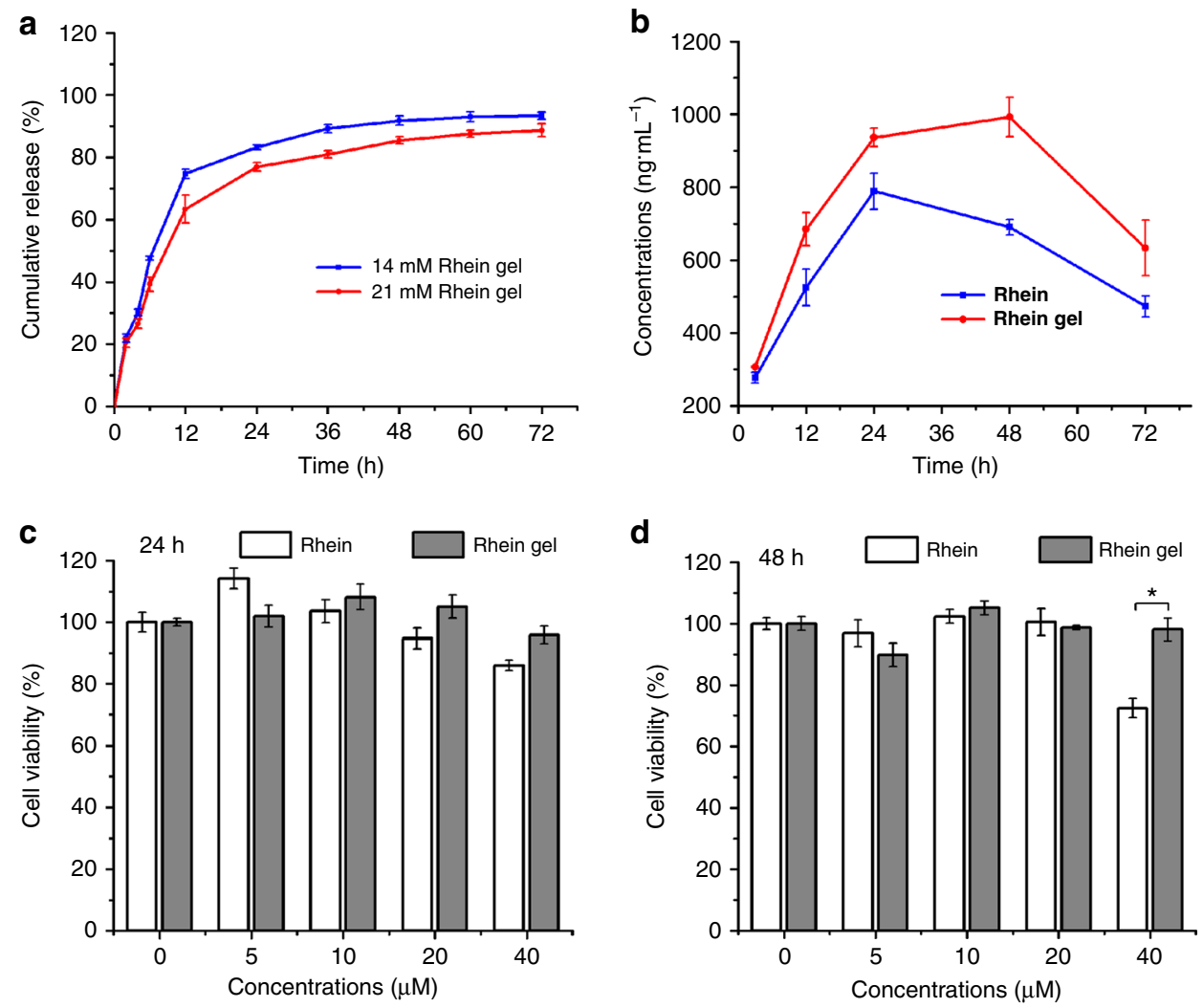

Fig. 4 Rhein hydrogel performed better sustainable release and lower cytotoxicity. a In vitro release profiles of rhein hydrogel, the concentrations of rhein hydrogel were $4 \mathrm{mg} \mathrm{mL}^{-1}(14.1 \mathrm{mM})$ and $6 \mathrm{mg} \mathrm{mL}^{-1}(21.1 \mathrm{mM})$, respectively. The gels were incubated with PBS buffer solution ( $\left.\mathrm{pH} 7.4\right)$ at $37^{\circ} \mathrm{C}$. $\mathbf{b}$ The curve of the concentration of rhein in the cells over time. c, $\mathbf{d}$ Cell viability ( $24 \mathrm{~h}$ and $48 \mathrm{~h}$ ) of BV2 cells incubated with different concentrations of rhein hydrogel and rhein, the concentrations of the samples range from 0 to $40 \mu \mathrm{M}$. Values are expressed as the means \pm SEM $(n=3)$. One-way ANOVA with Tukey's post hoc test. ${ }^{\star} p<0.05$. Error bars represent SEM

better inhibitions of p38 and PI3K/Akt phosphorylations induced by the rhein hydrogel were found compared to the free drug at 48 h. Our work demonstrates that the better therapeutic effects induced by the rhein hydrogel under sustained release are mainly associated with TLR4/NFKB inhibition.

\section{Discussion}

In this work, we have presented rhein-based direct self-assembly hydrogel. The rhein hydrogel exhibits excellent solubility, sustained release properties, low toxicity, and reversible stimuliresponsive properties to shear stress and temperature. As a directed self-assemble drug hydrogel, the rhein hydrogels significantly enhance anti-neuroinflammation compared with the free-drug through downregulation of proinflammatory cytokines and neurotoxic factors. The superior sustained release antineuroinflammatory effects from the rhein self-assembly hydrogels are associated with the inhibition of the TLR4/NFKB signaling pathway. The directed self-assemble hydrogels display superior solubility, optimal therapeutic efficacy, and fewer adverse effects. These properties facilitate the rhein hydrogel to serve as a potential anti-neuroinflammatory agent.

The gelation pathway is of key importance to append this unique gel system to other natural small molecules with low solubility. Our experiments indicate that the rhein hydrogel was formed by the self-assembly of rhein molecules through $\pi-\pi$ stacking, hydrogen bonds, and other non-covalent interactions. Based on the experimental results, a possible self-assembly mechanism of the rhein hydrogel was proposed. The evidence demonstrates that the rhein monomer is capable of accomplishing self-assembly processes without any structural modification or delivery carrier. This provides a potential guide for the self-assembly of other natural small molecules.

Understanding the anti-neuroinflammatory effects of the rhein hydrogel is the core of this study. It is notable that with treatment of free-drug and hydrogels for $24 \mathrm{~h}$, both drugs exhibit similar anti-neuroinflammatory effects without obvious cytotoxicity by reducing proinflammatory mediators, including TNF- $\alpha$, IL-6, IL12 , and iNOS. More interestingly, as the treatment continues for $48 \mathrm{~h}$, the rhein diminishes microglial cell viability at $40 \mu \mathrm{M}$, whereas the rhein hydrogel does not affect cell viability. Furthermore, the rhein hydrogel markedly lowers the levels of TNFa, IL-6, IL-12, and iNOS compared with the rhein monomer. The results reveal that the rhein hydrogel provides less cytotoxicity and better neuroinflammatory prevention than the free-drug under the sustained release control.

It is critical to explore how the rhein hydrogel interacts with protein targets to achieve the better therapeutic effects. Our work illustrates that both the rhein hydrogel and the free-drug block p38, PI3K/Akt, and TLR4/NFkB pathways to alleviate neuroinflammatory responses. Particularly, the better therapeutic effects induced by the rhein hydrogel under the sustained release are primarily associated with TLR4/NFkB inhibition, rather than $\mathrm{p} 38$ and PI3K/Akt pathways. This distinction may be attributed to the nanofiber structure of the rhein hydrogel. Moreover, we further use molecular docking techniques to elucidate the underlying mechanism. We confirm that TLR4 is a potential druggable target of rhein (Fig. 6a). This specific recognition facilitates the transfer of LPS to MD-2 protein, further activating the downstream components, including $\mathrm{NF \kappa B}$, and leading to the excess releases 
a
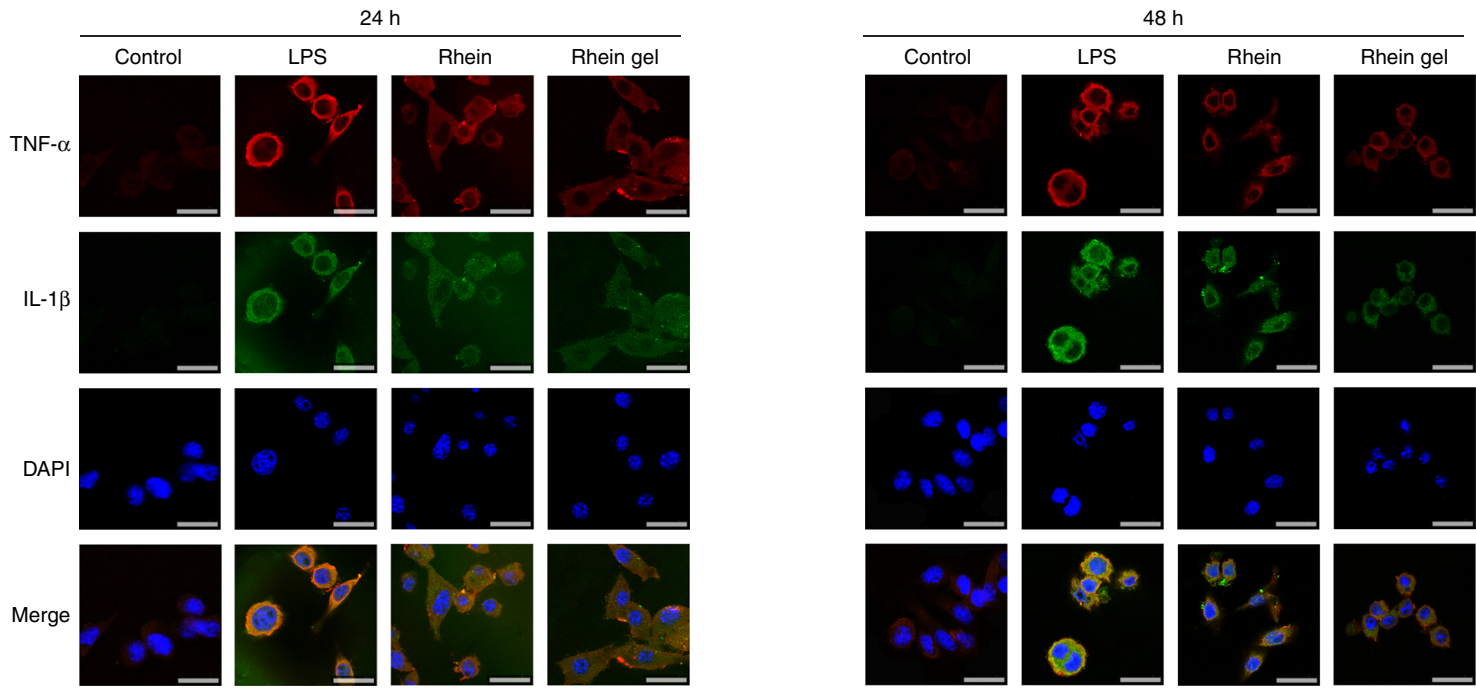

b
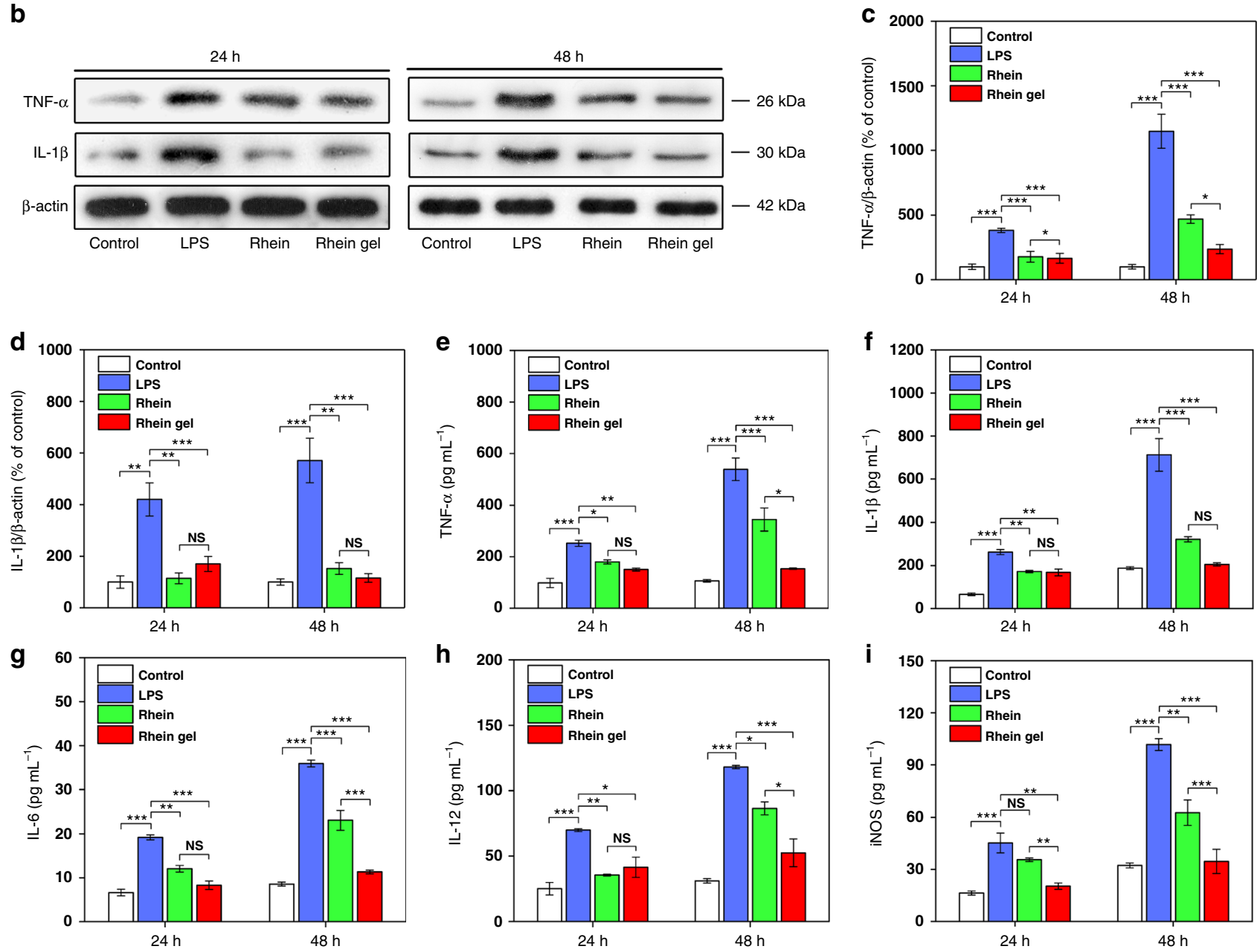

Fig. 5 Rhein and rhein hydrogel alleviated neuroinflammation in LPS-induced BV2 cells. a Confocal microscopy images from BV2 cells (treated with control, LPS, rhein and rhein hydrogel). Immunofluorescence staining is shown. Cell nuclei are shown in blue (DAPI), TNF- $\alpha$ is shown in red, IL-1 $\beta$ is shown in green, and yellow labeling represents co-localization. Scale bar, $15 \mu \mathrm{m}$. b Representative western blots of TNF- $\alpha$ and IL-1 $\beta$ proteins at $24 \mathrm{~h}$ and $48 \mathrm{~h}$. The uncropped and unprocessed scans of blots are presented in Supplementary Fig. 12. c Quantifications of TNF- $\alpha$ at $24 \mathrm{~h}$ and $48 \mathrm{~h}$. d Quantifications of IL-1 $\beta$ proteins at $24 \mathrm{~h}$ and $48 \mathrm{~h}$. e-i ELISA assays for determinations of TNF- $\alpha(\mathbf{e}), \mathrm{IL}-1 \beta(\mathbf{f}), \mathrm{IL}-6(\mathbf{g}), \mathrm{IL}-12(\mathbf{h})$ and iNOS (i) levels at $24 \mathrm{~h}$ and $48 \mathrm{~h}$. Values are expressed as means \pm SEM $(n=3)$. One-way ANOVA with Tukey's post hoc test. ${ }^{\star} p<0.05,{ }^{\star \star} p<0.01,{ }^{\star \star \star} p<0.001$. Error bars represent SEM 
a

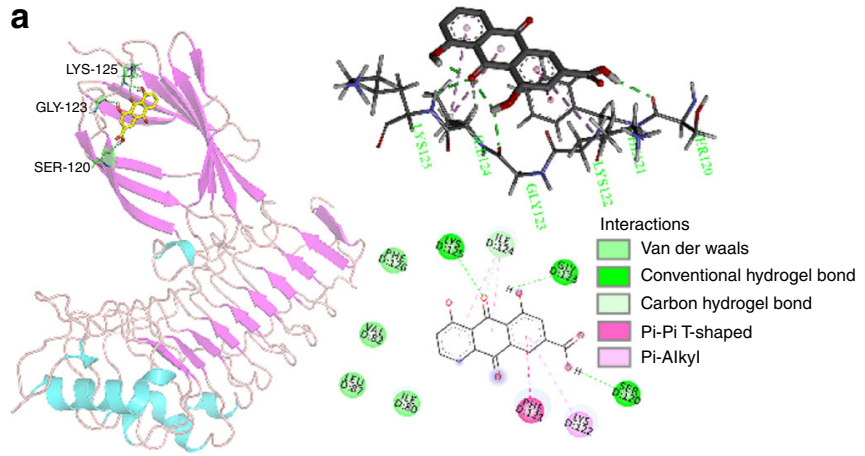

b

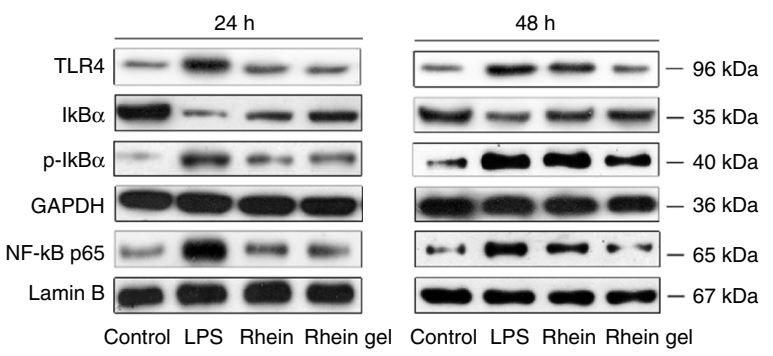

C

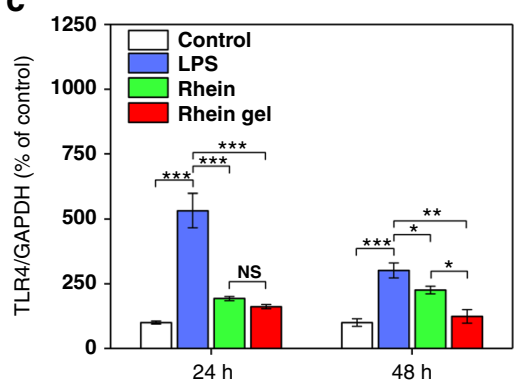

d

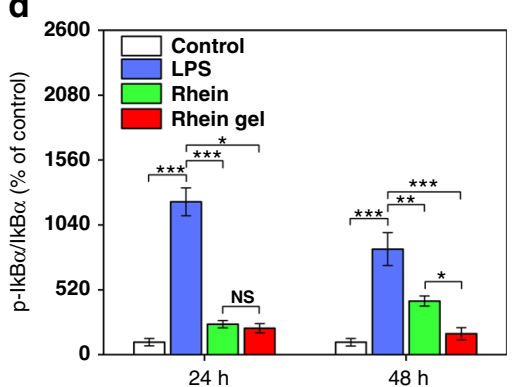

e

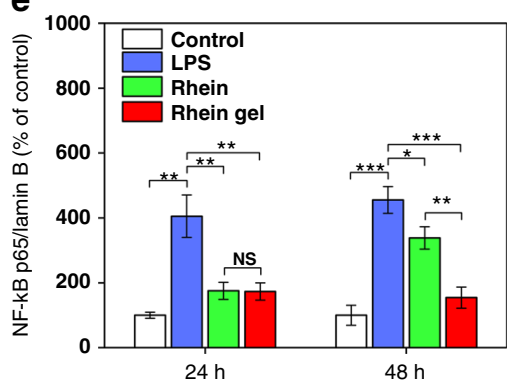

Fig. 6 Rhein and rhein hydrogel inhibited TLR4/NFKB pathway in LPS-induced BV2 cells. a Molecular docking analysis of the interaction between rhein and TLR4. Molecular docking model illustrated the interaction between rhein and the active sites of TLR4, showing the existences of intermolecular hydrogen bonds and $\pi-\pi$ interactions. $\mathbf{b}$ Rhein and its hydrogel blocked the TLR4/NFKB pathway, as shown by western blot analysis. Representative western blots of TLR4 in total proteins, $\mid \kappa B \alpha$ and $p-\mid \kappa B \alpha$ in cytosolic extracts, and p65 in nuclear extracts after treatment with rhein or rhein hydrogel for 24 and $48 \mathrm{~h}$. The

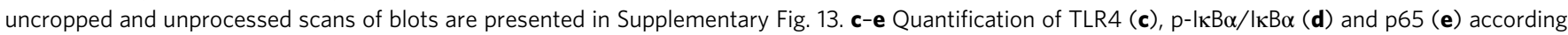
to densitometric analysis. The results are presented as a ratio of TLR4/GAPDH, $\mathrm{p}-\mathrm{I}_{\kappa} \mathrm{B} \alpha / \mathrm{I} \mathrm{KB} \alpha$ and $\mathrm{p} 65 / \mathrm{Lamin} \mathrm{B}$. Values are expressed as the means $\pm \mathrm{SEM}$ $(n=3)$. One-way ANOVA with Tukey's post hoc test. ${ }^{\star} p<0.05,{ }^{\star \star} p<0.01,{ }^{\star \star \star} p<0.001$. Error bars represent SEM

of proinflammatory cytokines and neurotoxic fators ${ }^{49-51}$. Therefore, the TLR4/MD-2/LPS complex represents a symmetrical manner to stimulate the proinflammatory responses. Inhibiting TLR4 dimerization is considered a key maneuver to regulate neuroinflammatory disorders ${ }^{52,53}$. This work reveals that strong hydrogen bonding and $\pi-\pi$ interactions exist between rhein and the residues of TLR4, including Ser120, Phe121, Gly123, Ile124, Lys125, and Lys122 (Fig. 6a). The rhein binds to MD-2 (subunit of TLR4 receptor) and forms a steric hindrance effect, which results in the inability of TLR4 to efficiently identify LPS, thereby inhibiting the NFKB signaling pathway. Compared to the free drug, rhein hydrogel consists of nanofibers with relatively larger size and orderly structure, which has a strong tendency to prolong the circulation time and allow for increased accumulation $^{54,55}$. On the other hand, the nanofibers are more easily uptaken by cells than the free-drug form ${ }^{56,57}$, providing more opportunity for these molecules to bind to TLR4. Furthermore, more hydrogen bonds and $\pi-\pi$ interactions from the aggregates contribute to the tight binding of TLR4. Hence, this hydrogel remarkably obscures the active site of TLR4 and blocks the access of the substrate, leading to better long-lasting effects and lower toxicity ${ }^{58}$. This illustration is in accord with the results of western blot in this study. Once LPS binds to TLR4 on the surface of microglia, it causes the translocation of p65 into the nucleus and binds to the DNA binding site. This regulates the transcription of its target genes, triggering the expression of pro-inflammatory enzymes and cytokines. The inhibition of TLR4 by the rhein nanofiber deactivates the downstream NFKB signaling pathway, leading to the downregulation of inflammatory mediators and cytokines. The present study shows that the superior inhibition of TLR $4 / \mathrm{NFKB}$ at $48 \mathrm{~h}$ may result from the slow dissolution and continuous release of the rhein molecules from the nanofibers. Accordingly, the rhein hydrogel can be used as a neuroinflammation-targeting agent.

In summary, we have explored direct self-assembly hydrogels of a natural product without any delivery carriers or structural modifications through hydrogen bonds, $\pi-\pi$ interactions and electrostatic interactions. The rhein hydrogel exhibits excellent stability, sustained release properties and reversible stimuliresponsive properties. The hydrogel consists of a 3D nanofiber network structure that prevents premature degradation. Moreover, it easily enters cells, leading to an intensive binding to the active site of toll-like receptor 4 . These advanced properties enable the rhein hydrogel to significantly dephosphorylate I $\mathrm{I} B \alpha$, inhibiting the nuclear translocation from the $\mathrm{p} 65$ at NFkB signaling pathway in LPS-induced BV2 microglia. In particular, the rhein hydrogel markedly alleviates neuroinflammation with a long-lasting effect without cytotoxicity compared with the equivalent free-drug dosing in vitro. To the best of our knowledge, this work provides a paradigm for discovering direct self-assembly hydrogels formed by natural small molecules to significantly promote therapeutic effects with no obvious cytotoxicity (Fig. 7).

\section{Methods}

Materials. Rhein was obtained by Xi'an Natural Field Bio-Technique Co., Ltd. Sodium bicarbonate $\mathrm{NaHCO}_{3}$ was provided from Sinopharm Chemical Reagent Co., Ltd. Phosphate buffer solutions (PBS) were purchased from Xiya Reagent (Chengdu, China). MTT reagent, dimethylsulfoxide (DMSO), destination access point identifier (DAPI), polyacrylamide and sodium dodecyl sulfonate were purchased from Sigma. Ultrapure water $(>18 \mathrm{M} \Omega)$ was used. All the reagents were used without further purification throughout this study.

Preparation of the rhein hydrogel. Rhein was dissolved in $\mathrm{NaHCO}_{3}(\mathrm{pH} 8.3$, $0.2 \mathrm{M}$ ) or PBS ( $\mathrm{pH} 8.0-9.4,0.1 \mathrm{M}$ ) followed by ultrasound or heat to obtain a homogeneous solution. Subsequently, the solution was cooled to room temperature 

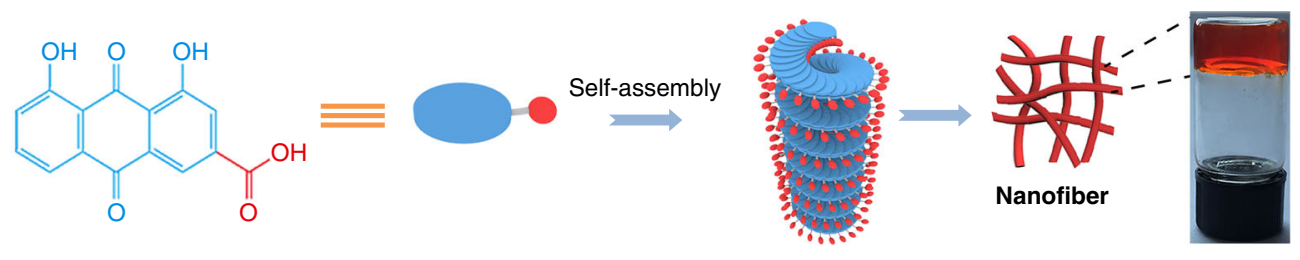

Rhein hydrogel

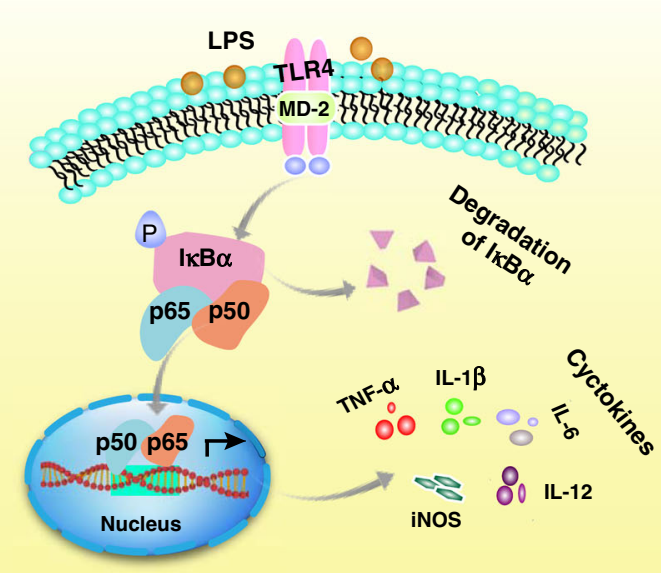

Inflammatory responses

Anti-inflammation

Fig. 7 Schematic depiction of neuroinflammatory prevention induced by rhein hydrogel. Rhein is self-assembled by non-covalent interaction to form nanofibers with left-handed, which are further crosslinked to form 3D network structure. When treating inflammatory BV2 cells with rhien nanofibers, the nanofibers slowly depolymerize to release rhein or rhein aggregates. They intensively bind to MD-2 (the subunit of TLR4 receptor), obscure the active site of TLR4 and further block the access of the substrate, leading to the inhibition of NFkB activation. Subsequently, the rhein hydrogel suppresses the release of neuroinflammatory factors and mediators

and accompanied by ultrasound. A uniform stable hydrogel was obtained after $5 \mathrm{~min}$, and the critical gel concentration was $4 \mathrm{mg} \mathrm{mL}^{-1}(14.1 \mathrm{mM})$.

Rheology test. The rheological studies were carried out on a rotated rheometer (AR 2000ex, TA Instrument, USA). The dynamic frequency sweep was measured at $0.1 \%$ strain, and the frequency was between 0.01 and $100 \mathrm{~Hz}$. The dynamic time sweep was measured at $0.1 \%$ strain, and the time was kept at $2400 \mathrm{~s}$. The straindependent oscillation was measured at $0.1-100 \%$ strain. The step-strain rheological experiment was performed in two steps. First, the rhein hydrogel was tested with a strain of $0.1 \%$ (step 1). Then, the strain was changed from 0.1 to $35 \%$ and remained at $35 \%$ for $1 \mathrm{~min}$ to completely damage the gels (step 2). Further, the strain was reduced from 35 to $0.1 \%$ again and remained at $0.1 \%$ for $3 \mathrm{~min}$ to observe whether the gel was restored. During the entire test, the frequency remained at $10 \mathrm{rad} \mathrm{s}^{-1}$ and the temperature was kept at $25^{\circ} \mathrm{C}$. All measurements were performed at $25^{\circ} \mathrm{C}$ and at a concentration of $5 \mathrm{mg} \mathrm{mL}^{-1}(17.6 \mathrm{mM})$ hydrogel.

Analysis of rhein aggregates by mass spectrometry. We studied the structure of rhein nanofibers by Ultrahigh-performance liquid chromatography and mass spectrometry (UHPLC-Q/Orbitrap/MS/MS). The UHPLC-Q-Orbitrap-MS system consisted of a Dionex U3000 UHPLC system (Thermo Fisher Scientific, MA, USA) and a high-resolution Q-exactive focus mass spectrometer (Thermo Fisher Scientific, Bremen, Germany). The Dionex U3000 UHPLC system was equipped with a quaternary Series RS pump, a degasser, an auto sampler, and a column compartment. The chromatographic system was coupled to the mass spectrometer with a heated electrospray ionization source (HESI). Xcalibur 3.0 (Thermo Fisher Scientific, Bremen, Germany) was used for UHPLC-MS control and data handling.

Chromatographic separation was performed on flowing injection at $35^{\circ} \mathrm{C}$. To obtain the most remarkable separation efficiency within a short time, the mobile phase, elution conditions, and other chromatographic parameters were optimized. Consequently, gradient elution was adopted using $\mathrm{H}_{2} \mathrm{O}(\mathrm{A})$ and methanol (B) as a mobile phase. The isocratic elution was programmed as follows: $0-5 \mathrm{~min}, 10-100 \%$ $\mathrm{B}$; The flow rate was $0.2 \mathrm{~mL} \mathrm{~min}^{-1}$, and the injection was $1.0 \mu \mathrm{L}$.

MS analysis: The HESI parameters were optimized for accurate mass measurement as follows: sheath gas flow rate, $30 \mathrm{~L} \mathrm{~min}^{-1}$; auxiliary gas flow rate, $8 \mathrm{~L} \mathrm{~min}{ }^{-1}$; spray voltage, $3.5 \mathrm{kV}$; capillary temperature, $320^{\circ} \mathrm{C}$; Slens RF level, $50 \mathrm{~V}$; auxiliary gas heater temperature, $120^{\circ} \mathrm{C}$; and collision energy, $35 \mathrm{eV}$. Nitrogen gas was used for spray stabilization, high energy collision dissociation, and damping gas in the C-trap. High-accuracy spectra were obtained in negative-ion mode. The mass range in the full scanning experiments was $200-3000 \mathrm{~m} / \mathrm{z}$.

FT-IR spectroscopy analysis. FT-IR spectra were recorded with a Perkin Elmer Spectrum One instrument (USA) and scanned between 4000 and $400 \mathrm{~cm}^{-1} . \mathrm{KBr}$ was mixed with the powdered samples to prepare the thin films. Materials were prepared at a concentration of $17.6 \mathrm{mM}$. $\mathrm{KBr}$ thin film was used as blank control

UV/Vis spectroscopy analysis. UV/Vis spectra were recorded on a Shimadzu UV-2450 spectrometer (Japan). We collected information from 800 to $200 \mathrm{~nm}$. Then $1 \mathrm{~mL}$ of the sample was detected by using a $10 \mathrm{~mm}$ aquartz cell.

Fluorescence spectroscopy analysis. The samples were detected using Hitachi F-7000 (Japan) in a $1 \mathrm{~mm}$ quartz cell. We prepared rhein solution and hydrogel with a concentration of $14.1 \mathrm{mM}$. Samples were excited at $450 \mathrm{~nm}$, monitoring the emission from 470 to $800 \mathrm{~nm}$.

X-ray powder diffraction test. XRD patterns were carried out at D/max 2550 power diffractometer (Japan) using a graphite-filtered $\mathrm{Cu} \mathrm{Ka}$ radiation manipulating at $40 \mathrm{kV}$. The sample was rhein xerogel.

Circular dichroism (CD) analysis. Circular dichroism spectra of different samples were obtained by using Jasco-815. Approximately $200 \mu \mathrm{L}$ of the sample was detected by using a $1 \mathrm{~mm}$ quartz cell. The bandwidth was set to $2 \mathrm{~nm}$ with a scanning speed of $100 \mathrm{~nm} \mathrm{~min}{ }^{-1}$. All scans were taken from 200 to $600 \mathrm{~nm}$, and all spectra were an average of 5 scans.

DFT calculation. The Gaussian 09 software package was used to perform geometry optimization and vibration analysis. The geometry optimization and vibration analyses were performed using M06-2X hybrid functional with all-electrons basis set $6-311 \mathrm{G}^{*}$. The vibration frequencies were evaluated at the same level of theory that of the optimization step.

In vitro drug release studies. First, $1 \mathrm{~mL}$ of hydrogel sample $(14.1 \mathrm{mM}$ and 21.1 $\mathrm{mM}$ ) with $1 \mathrm{~mL}$ of PBS buffer solution ( $\mathrm{pH} 7.4,0.01 \mathrm{M})$ was placed into separate dialysis bags (Viskase, MD25-2500, MW:2500) and incubated in $150 \mathrm{~mL}$ PBS buffer solution $(\mathrm{pH} 7.4,0.01 \mathrm{M})$ at $37^{\circ} \mathrm{C}$. Then, $10 \mathrm{~mL}$ of sample was removed 
at each time point and replenished $10 \mathrm{~mL}$ of fresh PBS solution, the sample was determined by UV/Vis absorption spectroscopy.

BV2 microglial cells culture. BV2 microglia cell lines (Catalogue Number: 3142C0001000000337) were purchased from National Infrastructure of Cell Line Resource (Beijing, China). BV2 microglial cells exhibit the phenotypic and the functional properties. They are the classic neuroinflammatory model. The immortalized mouse BV2 microglial cell line was purchased from National Infrastructure of Cell Line Resource (Beijing, China). The cells were grown and maintained in DMEM with $10 \% \mathrm{FBS}$ and antibiotics (1\% penicillin/streptomycin) under $5 \% \mathrm{CO}_{2}$ at $37^{\circ} \mathrm{C}$. The prepared cells were used for the subsequent experiments.

Determination of rhein concentration in BV2 cells by LC-MS. We used LC-MS to determine the concentration of rhein in the cells over time to assess the intracellular stability of rhein and rhein hydrogel. BV2 Cells were incubated in 6-well plates at a density of $2 \times 10^{6}$ cells for $12 \mathrm{~h}$. We prepared the DMEM containing $15 \mu \mathrm{M}$ of rhein and rhein hydrogel, respectively, and $2 \mathrm{~mL}$ of DMEM was added to cells. The cells were then incubated for $3 \mathrm{~h}, 12 \mathrm{~h}, 24 \mathrm{~h}, 48 \mathrm{~h}$, and $72 \mathrm{~h}$, respectively. Subsequently, the DMEM containing drugs was removed. $500 \mu \mathrm{L}$ of methanol was added to each well to lyse the cells and release the compounds. The sample was collected and centrifuged at $22,000 \times g$ for 20 min after treated with a vortex shaker for $5 \mathrm{~min}$. The obtained sample was detected by LC-MS.

Cell viability measurement. Cells were seeded in 96 -well plates $\left(1 \times 10^{4} \mathrm{~mL}^{-1}\right.$ cells). The cells were then treated with a series of concentrations of drugs for $12 \mathrm{~h}$ Then, $10 \mu \mathrm{L}$ of MTT reagent $\left(5 \mathrm{mg} \mathrm{mL}^{-1}\right.$, Sigma) was added to each well and incubated for $4 \mathrm{~h}$. Afterward, the supernatants were removed. Next, $150 \mu \mathrm{L}$ of DMSO was added to dissolve the formazan crystals. The absorbance was measured with a microplate reader (Huisong Technology Development Co., Ltd, Shenzhen, China).

Immunofluorescence staining. After various treatments, BV2 cells were fixed in $4 \%$ paraformaldehyde and then permeabilized with $0.3 \%$ Triton X-100. The cells were blocked with $5 \%$ bovine serum albumin (BSA). Subsequently, the cells were immunostained with primary antibodies, including TNF- $\alpha$ (Proteintech, 60291-1Ig, mouse, 1:50) and IL-1 $\beta$ (Proteintech, 16806-1-AP, rabbit, 1:50). After incubation at $4{ }^{\circ} \mathrm{C}$ overnight, the cells were further incubated with the corresponding Alexa fluor 594-conjugated goat anti-mouse IgG (Proteintech, SA00013-3, 1:500) or Alexa fluor 488-conjugated goat anti-rabbit IgG (Proteintech, SA00013-2, 1:500) for $1 \mathrm{~h}$ at room temperature. Finally, nuclei were counterstained with $4^{\prime}, 6$-diamidino-2-phenylindole (DAPI, Sigma). All images were captured using a confocal microscope (TCS SP8 X \& MP, Leica, Germany).

ELISA method. BV2 cells $\left(1 \times 10^{5}\right.$ cells per well in a 24 -well plate) were pretreated with treatments for $1 \mathrm{~h}$ and stimulated with LPS $\left(100 \mathrm{ng} \mathrm{mL}^{-1}\right)$. After treatment for $24 \mathrm{~h}$ and $48 \mathrm{~h}$, the supernatants were collected. The concentrations of TNF- $\alpha$, IL- $1 \beta$, IL-6, IL-12, and iNOS in the culture medium were measured by ELISA kits, following the procedures recommended by the supplier (Cusabio, Wuhan, China).

Western blot assay. Total cellular and nuclear proteins were extracted from BV2 microglia cells. The protein concentrations were determined by BCA protein assay kit. Proteins were electrophoresed on 10\% SDS-polyacrylamide gels, and transferred onto a PVDF membrane (Millipore, Bedford, MA). The membranes were blocked in 5\% skim milk with 20\% Tween20 (TBST) for $1 \mathrm{~h}$ at room temperature. We next used the following primary antibodies and dilutions: TNF- $\alpha$ (Abcam, ab66579, rabbit, 1:1000), IL-1 3 (Proteintech, 16806-1-AP, rabbit, 1:50), TLR4 (Proteintech, 19811-1-AP, rabbit, 1:500), IKBa (Abcam, ab32518, rabbit, 1:1000), p-IKBa (Cell Signaling Technology, \#9246, mouse, 1:1000), p65 (Cell Signaling Technology, \#6956, mouse, 1:1000), p38 (Proteintech, 14064-1-AP, rabbit, 1:2000), p-p38 (Abcam, ab195049, rabbit, 1:1000), PI3K (Abcam, ab40755, rabbit, 1:1000), p-PI3K (Cell Signaling Technology, \#4228, rabbit, 1:1000), Akt (Cell Signaling Technology, \#9272, rabbit, 1:1000), p-Akt (Cell Signaling Technology, \#4060, rabbit 1:2000), $\beta$-actin (Proteintech, 60008-1-Ig, mouse, 1:4000), GAPDH (Bioworld, AP0063, rabbit, 1:2000) and Lamin B (Proteintech, 66095-1-Ig mouse, 1:5000). The blots were incubated with the primary antibodies overnight at $4{ }^{\circ} \mathrm{C}$. After washing with TBST, the anti-mouse secondary antibodies (Proteintech, 1:3000, SA00001-1) and the anti-rabbit secondary antibodies (Proteintech, 1:6000, SA00001-2) were incubated for $1 \mathrm{~h}$ at room temperature. The proteins were visualized by an enhanced chemiluminescence detection system (ThermoFisher Scientific, MA, USA). The uncropped and unprocessed scans of blots are presented in Supplementary Figs. 12 and 13

Molecular docking studies. Autodock 4.0 was used in this study to evaluate the potential molecular binding mode, the PyMol molecular viewer (http://www. pymol.org/) was employed to analyze the docked structures. The crystal structure of the TLR4-MD-2 Complex with bound endotoxin antagonist Eritoran (PDB code: 2Z65) was downloaded from the RCSB Protein Data Bank (www.rcsb.org). The 3D chemical structure of rhein was retrieved from PubChem compound database (NCBI, USA) and was subjected to minimized the energy by using molecular mechanics-2 (MM2) force field in Chem 3D Pro. The protein-ligand docking active site was defined by the location of the original ligand (Eritoran).
Lamarckian Genetic Algorithm (LGA) was used and dimensions of the grids were set at $60 \times 60 \times 60 \AA$ in the $\mathrm{x}, \mathrm{y}$ and $\mathrm{z}$ directions, with a spacing of $0.375 \AA$ between the grid points and the center placed at the active site of TLR4-MD-2 Complex crystallographic structures. All other docking and consequent scoring parameters used were kept at their default settings. The docked conformation corresponding to the lowest binding energy was selected as the most probable binding conformation.

Statistical analysis. The results for statistical analysis are presented as the mean \pm standard error mean (SEM). The statistical differences between groups were tested by one-way analysis of variance (ANOVA) for multiple comparisons followed by Tukey's post hoc test using GraphPad Prism software (version 7.0, La Jolla, CA, USA). $P$-value $<0.05$ was considered statistically significant $\left({ }^{*} p<0.05,{ }^{* *} p<0.01\right.$, $\left.{ }^{* * *} p<0.001\right)$

\section{Data availability}

The authors declare that all data which supports the findings are provided with the paper and its supplementary information. All data is available from the corresponding authors upon reasonable request.

Received: 2 September 2018 Accepted: 19 March 2019

Published online: 08 April 2019

\section{References}

1. Harvey, A. L., Edradaebel, R. \& Quinn, R. J. The re-emergence of natural products for drug discovery in the genomics era. Nat. Rev. Drug. Discov. 14, 111-129 (2015).

2. Newman, D. J. \& Cragg, G. M. Natural products as sources of new drugs from 1981 to 2014. J. Nat. Prod. 79, 629-661 (2016).

3. Wang, M., Cheetham, A. G. \& Cui, H. Building nanostructures with drugs. Nano Today 11, 13-30 (2016).

4. Lam, F. C. et al. Enhanced efficacy of combined temozolomide and bromodomain inhibitor therapy for gliomas using targeted nanoparticles. Nat. Commun. 9, 1991-2001 (2018).

5. Tibbitt, M. W., Dahlman, J. E. \& Langer, R. Emerging frontiers in drug delivery. J. Am. Chem. Soc. 138, 704-717 (2016).

6. Webber, M. J. \& Langer, R. Drug delivery by supramolecular design. Chem. Soc. Rev. 46, 6600-6620 (2017)

7. Li, J. et al. D-amino acids boost the selectivity and confer supramolecular hydrogels of a nonsteroidal anti-Inflammatory drug (NSAID). J. Am. Chem. Soc. 135, 542-545 (2013).

8. Pappas, C. G. et al. Dynamic peptide libraries for the discovery of supramolecular nanomaterials. Nat. Nanotech. 11, 960-967 (2016)

9. Brown, T. E. \& Anseth, K. S. Spatiotemporal hydrogel biomaterials for regenerative medicine. Chem. Soc. Rev. 46, 64-70 (2017).

10. Hu, B. et al. Supramolecular hydrogels for antimicrobial therapy. Chem. Soc. Rev. 27, 6917-6929 (2018).

11. Wang, H., Feng, Z. \& Xu, B. Bioinspired assembly of small molecules in cell milieu. Chem. Soc. Rev. 46, 2421-2436 (2017).

12. Liu, Q. et al. A supramolecular shear-thinning anti-Inflammatory steroid hydrogel. Adv. Mater. 28, 6680-6686 (2016).

13. Zhao, F., Ma, M. L. \& Xu, B. Molecular hydrogels of therapeutic agents. Chem Soc. Rev. 38, 883-891 (2009).

14. Adlerabramovich, L. et al. Phenylalanine assembly into toxic fibrils suggests amyloid etiology in phenylketonuria. Nat. Chem. Biol. 8, 701-706 (2012).

15. Wang, J. et al. Enzymatic formation of curcumin in vitro and in vivo. Nano. Res. 11, 1-9 (2018)

16. Yuan, G. et al. Enzyme-instructed molecular self-assembly confers nanofibers and a supramolecular hydrogel of taxol derivative. J. Am. Chem. Soc. 131, 13576-13577 (2009).

17. Wang, H. et al. The inhibition of tumor growth and metastasis by selfassembled nanofibers of taxol. Biomaterials. 33, 5848-5853 (2012).

18. Cheetham, A. G., Zhang, P., Lin, Y., Lock, L. L. \& Cui, H. Supramolecular nanostructures formed by anticancer drug assembly. J. Am. Chem. Soc. 135, 2907-2910 (2013).

19. Tang, W., Yang, J., Zhao, Z., Lian, Z. \& Liang, G. Intracellular coassembly boosts the anti-inflammation capacity of dexamethasone. Nanoscale $\mathbf{9}$, 17717-17721 (2017)

20. Tomasini, C. \& Castellucci, N. ChemInform abstract: peptides and peptidomimetics that behave as low molecular weight gelators. Chem. Soc. Rev. 44, 156-172 (2013)

21. Yang, W. et al. Rhein and rhubarb similarly protect the blood-brain barrier after experimental traumatic brain injury via gp9lphox subunit of NADPH oxidase/ ROS/ERK/MMP-9 signaling pathway. Sci. Rep. 6, 37098-37021 (2016).

22. Liu, J. et al. Rhein lysinate decreases the generation of $\beta$-amyloid in the brain tissues of Alzheimer's disease model mice by inhibiting inflammatory response and oxidative stress. J. Asian. Nat. Prod. 15, 756-763 (2013). 
23. $\mathrm{Xu}, \mathrm{X}$. et al. Rhein exhibits antioxidative effects similar to Rhubarb in a rat model of traumatic brain injury. BMC. Complem. Altern. Med. 17, 140-149 (2017).

24. He, L. N. et al. Reactive metabolite activation by CYP2C19-mediated rhein hepatotoxicity. Xenobiotica 45, 361-372 (2015).

25. Wu, W., Yan, R., Yao, M., Zhan, Y. \& Wang, Y. Pharmacokinetics of anthraquinones in rat plasma after oral administration of a rhubarb extract. Biomed. Chromat. 28, 564-572 (2014).

26. Gómez-Gaete, C. et al. Development, characterization and in vitro evaluation of biodegradable rhein loaded microparticles for treatment of osteoarthritis. Eur. J. Pharm. Sci. 96, 390-397 (2016).

27. Rizwanullah, M., Ahmad, J. \& Amin, S. Nanostructured lipid carriers: a novel platform for chemotherapeutics. Curr. Drug. Deliv. 13, 4-26 (2016).

28. Jin, H. et al. Tumor ablation and therapeuticimmunity induction by an injectable peptide hydrogel. Acs Nano 12, 3295-3310 (2018).

29. Sun, Z. et al. Multistimuli-responsive, moldable supramolecular hydrogels cross-linked by ultrafast complexation of metal Ions and biopolymers. Angew. Chem. Int. Ed. Engl. 127, 7944-7948 (2015).

30. Lyu, D., Chen, S. \& Guo, W. Liposome crosslinked polyacrylamide/DNA hydrogel: a smart controlled-release system for small molecular payloads. Small. 14, 1704039-1704046 (2018).

31. Mealy, J. E. et al. Injectable granular hydrogels with multifunctional properties for biomedical applications. Adv. Mater. 30, 1705912-1705917 (2018).

32. Ikeda, M. et al. Installing logic-gate responses to a variety of biological substances in supramolecular hydrogel-enzyme hybrids. Nat. Chem. 6, 511-518 (2014).

33. Wang, X. et al. A Co(2+)-selective and chirality-sensitive supermolecular metallohydrogel with a nanofiber network skeleton. Nanoscale 8, 6479-6483 (2016).

34. Young, L. M. et al. Screening and classifying small-molecule inhibitors of amyloid formation using ion mobility spectrometry-mass spectrometry. Nat. Chem. 7, 73-81 (2015).

35. Yuanjiao, L. et al. Mass spectrometric and spectrophotometric analyses reveal an alternative structure and a new formation mechanism for melanin. Anal. Chem. 87, 7958-7963 (2015)

36. Irwansyah, I. et al. Gram-positive antimicrobial activity of amino acid-based hydrogels. Adv. Mater. 27, 648-654 (2015).

37. Wang, X. et al. Designing isometrical gel precursors to identify the gelation pathway for nickel-selective metallohydrogels. Dalton. T. 45, 18438-18442 (2016).

38. Sun, Z. et al. Ferrocenoyl phenylalanine: a new strategy toward supramolecular hydrogels with multistimuli responsive properties. J. Am. Chem. Soc. 135, 13379-13386 (2013).

39. Yao, H., Domoto, K., Isohashi, T. \& Kimura, K. In situ detection of birefringent mesoscopic $\mathrm{H}$ and $\mathrm{J}$ aggregates of thiacarbocyanine dye in solution. Langmuir 21, 1067-1073 (2005).

40. Ishikawa, K., Kameta, N., Masuda, M., Asakawa, M. \& Shimizu, T. Boroxine nanotubes: moisture-sensitive morphological transformation and guest release. Adv. Funct. Mater. 24, 603-609 (2014).

41. Yuan, W. Z. et al. Changing the behavior of chromophores from aggregationcaused quenching to aggregation-induced emission: development of highly efficient light emitters in the solid state. Adv. Mater. 22, 2159-2163 (2010).

42. Liu, K. et al. Coordination-triggered hierarchical folate/zinc supramolecular hydrogels leading to printable biomaterials. Acs Appl. Mater. Interfaces 10, 4530-4539 (2018).

43. Liu, G. et al. Controlling supramolecular chirality of Two-component hydrogels by J- and $\mathrm{H}$-aggregation of building blocks. J. Am. Chem. Soc. 140, 6467-6473 (2018).

44. Xu, H. et al. Supramolecular nanofibers of curcumin for highly amplified radiosensitization of colorectal cancers to Ionizing radiation. Adv. Funct. Mater. 28, 1707140-1707150 (2018).

45. Hennessy, E., Griffin, É. W. \& Cunningham, C. Astrocytes are primed by chronic neurodegeneration to produce exaggerated chemokine and cell Infiltration responses to acute stimulation with the cytokines IL- $1 \beta$ and TNF- $\alpha$. J. Neurosci. 35, 8411-8422 (2015).

46. Fan, H. H. et al. Hyperoside inhibits lipopolysaccharide-induced inflammatory responses in microglial cells via $\mathrm{p} 38$ and NFkB pathways. Int. Immunopharmac. 50, 14-21 (2017).

47. Jung, J. S. et al. Suppression of LPS-induced neuroinflammation by morin via MAPK, PI3K/Akt, and PKA/HO-1 signaling pathway modulation. J. Agr. Food. Chem. 65, 373-382 (2016).

48. Rahimifard, M. et al. Targeting the TLR4 signaling pathway by polyphenol: A novel therapeutic strategy for neuroinflammation. Ageing Res. Rev. 36, 11-19 (2017).

49. Lu, Y. C., Yeh, W. C. \& Ohashi, P. S. LPS/TLR4 signal transduction pathway. Cytokine 42, 145-151 (2008).
50. Zhu, J. et al. Involvement of mitogen-activated protein kinase and NF-kB signaling pathways in perfluorooctane sulfonic acid-induced inflammatory reaction in BV2 microglial cells. J. Appl. Toxicol. 35, 1539-1549 (2015).

51. Lan, X. et al. Pinocembrin protects hemorrhagic brain primarily by inhibiting toll-like receptor 4 and reducing M1 phenotype microglia. Brain Behav. Immun. 61, 326-339 (2017).

52. Gao, H. et al. Total tanshinones exhibits anti-inflammatory effects through blocking TLR4 dimerization via the MyD88 pathway. Cell Death Dis. 8, e3004 (2017).

53. Lan, X., Han, X., Li, Q., Yang, Q. W. \& Wang, J. Modulators of microglial activation and polarization after intracerebral haemorrhage. Nat. Rev. Neurol. 13, 420-433 (2017)

54. Cui, H. \& Xu, B. Supramolecular medicine. Chem. Soc. Rev. 46, 6430-6432 (2017).

55. Cheetham, A. G., Chakroun, R. W., Ma, W. \& Cui, H. Self-assembling prodrugs. Chem. Soc. Rev. 46, 6638-6663 (2017).

56. Yanbin, C. et al. Environment-sensitive fluorescent supramolecular nanofibers for imaging applications. Anal. Chem. 86, 2193-2199 (2014).

57. Yang, C. et al. A supramolecular hydrogelator of curcumin. Chem. Commun 50, 9413-9415 (2014).

58. Dong, J. et al. Baicalin inhibits the lethality of ricin in mice by inducing protein oligomerization. J. Biol. Chem. 290, 12899-12907 (2015).

\section{Acknowledgements}

This work was supported by the National Natural Science Foundation of China (Nos. 81303074, 81673719, 21473257, and 21773311), the Outstanding Youth Foundation of Hunan Provincial Natural Science Foundation of China (No. 2019JJ30042), the Hunan Provincial Natural Science Foundation of China (No. 2019JJ50960), and the Special Program for Applied Research on Super Computation of NSFC-Guangdong Joint Fund. We are very grateful to Prof. Zhimou Yang from NanKai University for giving us guidance on experimental methods of using LC-MS to evaluate the stability of rhein gel in cells.

\section{Author contributions}

Y.W., Y.Z., R.F. and T.T. conceived and designed the research. J.Z. prepared rhein hydrogel and investigated the self-assembly mechanism of the rhein hydrogel. R.F., Y.Y., H.Y., J.L., P.Z. and P.G. conducted the study of cytotoxic and anti-inflammatory mechanisms of rhein hydrogel. H.W., T.Y. and L.D. carried out the DFT theoretical calculation. J.Z. performed the spectroscopic characterizations. L.Y. and L.R. performed mass experiments. Z.S. prepared and performed data collection. J.Z., Y.W., R.F. and Y.Y. and drafted and revised the manuscript. J.Z., Y.Z. and Y.W. wrote Supplementary information.

\section{Additional information}

Supplementary Information accompanies this paper at https://doi.org/10.1038/s41467019-09601-3.

Competing interests: The authors declare no competing interests.

Reprints and permission information is available online at http://npg.nature.com/ reprintsandpermissions/

Journal peer review information: Nature Communications thanks the anonymous reviewers for their contribution to the peer review of this work. Peer reviewer reports are available

Publisher's note: Springer Nature remains neutral with regard to jurisdictional claims in published maps and institutional affiliations.

Open Access This article is licensed under a Creative Commons Attribution 4.0 International License, which permits use, sharing, adaptation, distribution and reproduction in any medium or format, as long as you give appropriate credit to the original author(s) and the source, provide a link to the Creative Commons license, and indicate if changes were made. The images or other third party material in this article are included in the article's Creative Commons license, unless indicated otherwise in a credit line to the material. If material is not included in the article's Creative Commons license and your intended use is not permitted by statutory regulation or exceeds the permitted use, you will need to obtain permission directly from the copyright holder. To view a copy of this license, visit http://creativecommons.org/ licenses/by/4.0/.

(C) The Author(s) 2019 